\title{
Role of Closed Reduction and Percutaneous Fixation for Intraarticular Fractures of Calcaneum in Adults.
}

\author{
Dr.Vivek A. Patel ${ }^{1}$, Dr.Ramesh Panchal ${ }^{2}$, Dr. Parth Trivedi ${ }^{3}$ \\ ${ }^{I}$ Assistant Professor of orthopedics, Department of orthopedics, Pramukh Swami medical college and Shree \\ Krishna hospital, Karamsad,Anand, Gujarat,India. \\ ${ }^{2}$ Professor \& Head of the Department, Department of orthopedics, Pramukh Swami medical college and Shree \\ Krishna hospital, Karamsad, Anand, Gujarat,India. \\ ${ }^{3}$ Post Graduate,Department of orthopaedics,Pramukh Swami medical college and shree Krishna \\ hospital,Karamsad,Anand, Gujarat,India.
}

\begin{abstract}
Calcaneum is most commonly fractured tarsal weight bearing bone. No controversy in the management of extraarticular Calcaneum fractures because they do well in terms of functional recovery and fracture healing; but as far as Intraarticular fractures of calcaneum concerned (about $75 \%$ of all calcaneum fractures \& most of the patients are young) no clear consensus exists on treatment protocol for this class. As a traditional thought whether the joint is reconstructable or not; has great bearing on patients propensity to develop subtalar arthritis and later on fusion. Our study shows good results as far as functional outcome concerned with closed reduction \& Percutaneous fixation by pins and screws in compare to traditional approaches of treatment like open reduction \& in some cases conservative cast immobilization where high complication rates prevails.
\end{abstract}

Key Words: Closed Reduction, Percutaneous Fixation, Calcaneum.

\section{Introduction}

The calcaneus is the most frequently fractured of the tarsal bones, and represents $1 \%$ to $2 \%$ of all fractures. Calcaneus fractures can be broadly classified into intra-articular and extra-articular types. Patients with extraarticular calcaneus fracture generally do well in terms of functional recovery and fracture healing. Even when there is a displacement at the fracture site, fracture union is almost universal and when early movement is implemented, joint stiffness has been shown to be a minimal long-term problem.

Intra-articular fractures are more common, representing $75 \%$ of all calcaneal fractures, with spectrum of fragment patterns. Typically, they are the result of high-energy impact, with mechanisms of injury including falls from height and motor vehicle accidents. As such, they tend to be more common in young laborers who work on scaffolding, bridges, and ladders. These injuries can result in considerable long-term impairment and thus can be career-ending in a young, otherwise healthy individual. They represent a significant therapeutic challenge, and despite their frequency, no clear consensus exists on treatment protocol for this class of fractures.

One of the prognostic indicators of the outcome of a calcaneus fracture is the amount of injury to the posterior facet ${ }^{1}$. Whether this joint is reconstructable has great bearing on a patient's propensity to develop significant arthritis, a condition that may lead to a subtalar fusion.

Healing is a biological process helped by mechanical stability. In $21^{\text {st }}$ century the focus has shifted from anatomical fixation to biological fixation of all fractures. Closed reduction and percutaneous pinning in its various forms has been advocated only for specific types of fractures such as the tongue type pattern of Essex Lopressti ${ }^{2}$. Recently results of a multi-centered prospective trial suggested that the differences between ORIF and closed treatment might be less than previously thought ${ }^{3,4,5,6,7}$.If the articular reduction might be less important than restoration of hind foot architecture, then restoring bony alignment without formal ORIF may avoid the complications of ORIF, while also accomplishing many of the treatment goals. The present report describes our preliminary experience with a specific technique of closed reduction and per cutaneous fixation in a consecutive series of intra-articular calcaneus fractures.

\section{Material \& Methods}

We have treated 20 patients and 22 fractures ( 2 patients had bilateral calcaneus fractures) of intraarticular calcaneus fractures by closed reduction and percutaneous fixation by ST pin and/ or K-wire and/ or CC screws at our institute between January -2008 to April- 2009.

Radiographic evaluation includes three views: lateral view of the calcaneum, harris axial view and intra-operatively broadens view to evaluate the posterior facet. Bohler's angle and Gissane's angle evaluated in lateral and varus in axial view evaluated. Computed tomography some times used to evaluate the posterior facet 8 and the fracture comminution to assist in pre-oprative planning. Intra-operatively contra lateral calacaneus 
Bohler's angle $\mathrm{e}^{9,10,11}$ and Gissane's angle ${ }^{12}$ evaluated to gauge for significant deformity from the patient's normal baseline.

Each case has been followed till clinico-radiological union and subsequent follow-up after 6 to 12 months post-operatively. In the study of 20 patients $95 \%$ cases were males $5 \%$ cases were females. All were closed fractures. $80 \%$ cases were aged between $22-40$ years. $15 \%$ of cases were between $40-60$ years and $5 \%$ cases were above 60 years. $70 \%$ of cases have history of fall from height; more than 5 feet and $30 \%$ of cases sustained road traffic accidents. 30\% cases had tongue type fractures and $70 \%$ cases had joint depression type fractures. After fixation majority of the patients were splinted with posterior plaster splint for atleast 4 weeks depending upon intraoperative stability of reduction. Patients were followed-up every 4 weeks till fracture union and then when we called them 6-12 months post operatively 18 cases were contactable.

\section{Operative Technique}

In the operative technique patient was given supine position at the edge of the table and manual compression across the calcaneus was applied to reduce the width under IITV guidance. A large diameter ST pin was placed across the calcaneus and axial traction was given to distract the talo-calcaneal joint and by that varus valgus alignment achieved. By percutaneous method back of ST pin or push screw was used to elevate the talus $\&$ distract the talo-calcaneal joint and elevate the posterior calcaneal facet. In the later and axial view 1 or 2 ; $6.5 \mathrm{~mm} \mathrm{CC}$ screws were passed from lateral aspect of the calcaneum into the sustentaculum tali of calcaneum to reduce the width of depressed and fractured calcaneum. In axial and lateral views 1 or 2; ST pins were passed in the axis of calcaneum as far as possible in a crossed manner to stabilize the juxta chondral part of bone of posterior facet (Fig.).

Fig:
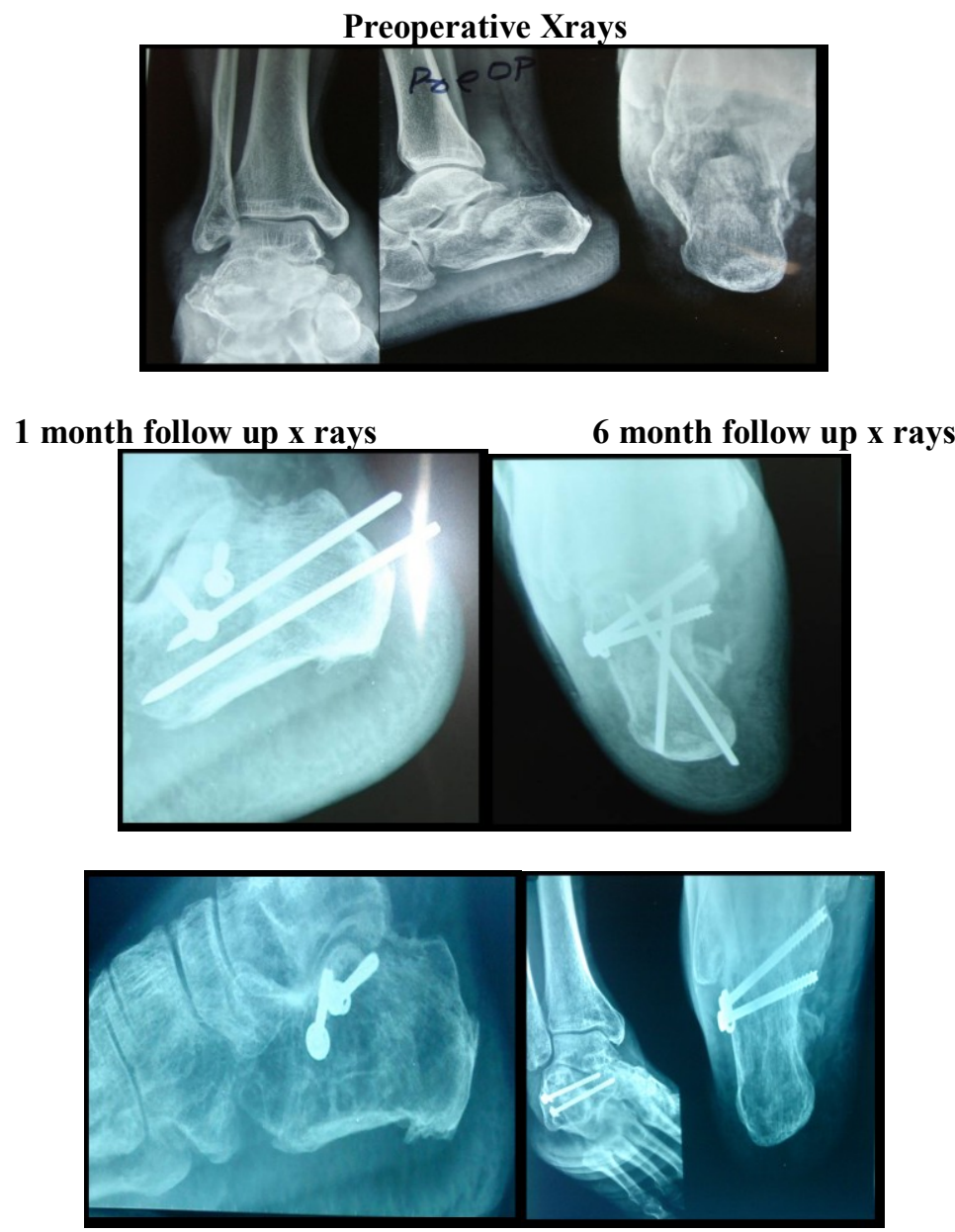

IV. Results

All the 20 patients were come for follow up up to fracture union. Three patients had associated injuries typical of polytraumatised patients. Average injury to operative time interval was 3.5 days (min. $2 \&$ max. 6 days).Avg. hospital stay was 5 days post surgery (min. $2 \&$ max. 10 days).Partial wt. bearing started avg. at 8 
wks, Full wt bearing at 12 weeks and average ankle mobilization started at 10 weeks (min. at immediate post operatively to max. 12 wks; depending upon intraoperative stability of reduction). $\mathrm{K}$ wires removed at average of 10.5 wks. Clinico radiological union at average of 11 wks. Post op. average Bohler's angle 25.6 degree and average Gissane's angle 122 degree noted. No clinically significant varus or valgus in any cases(less than 5 degrees in any cases). Average AOFAS score ${ }^{13}$ was 79.

Then when we called them 6-12 months post operatively 18 cases were contactable and came for evaluation with radiological consolidated fracture \& angle same as stated above in lateral and axial view.These patients were all satisfied with their present condition and felt they had an excellent result (TABLE).No patient had infection. One patient had exposed tendo calcaneus $6 \mathrm{wks}$ post operatively on which reverse sural flap was done and later on satisfied. Three (Out of those three; two patients had peroneal tendonitis quite relieved by conservative treatment,one patient had loss of calcaneal height \& is developing subtalar arthrosis.) patients had moderate discomfort with full activity, were wearing normal shoes with inserts and had no limp. One patient had severe pain with activity and had difficulties in normal shoe wear with loss of height of calcaneum \& subtalar arthrosis. So far, none of the patient had subtalar fusion.

Table:

\begin{tabular}{|l|l|l|l|l|l|l|}
\hline \multirow{2}{*}{ Outcome } & \multicolumn{3}{|c|}{ Bohler's Angle } & \multicolumn{2}{c|}{ Total } \\
\cline { 2 - 6 } & $<20$ Degree & \multicolumn{2}{|c|}{$>\mathbf{2 0}$ Degree } & \multicolumn{2}{c|}{} \\
\hline & $\mathbf{N}$ & $\mathbf{\%}$ & $\mathbf{N}$ & $\mathbf{\%}$ & $\mathbf{N}$ & $\%$ \\
\hline $\begin{array}{l}\text { Excellent+ } \\
\text { Good }\end{array}$ & 01 & 5.55 & 12 & 66.66 & 13 & 72.22 \\
\hline Fair + Poor & 03 & 16.67 & 02 & 11.11 & 05 & 27.78 \\
\hline Total & 04 & 100 & 14 & 100 & 18 & 100 \\
\hline
\end{tabular}

\section{Discussion}

Closed Reduction \& percutaneous fixation of intraarticular calcaneum fractures gives biological environment with optimal mechanical stability with early permitted mobilization.It takes care of varus / valgus alignment, width \& as far as possible the pitches of the calcaneum with significantly reduced chance of malunion.

Complications can occur regardless of the strategy chosen. These includes wound healing problems, infection, neurological complications, peroneal tendon injury, thromboembolism,compartment syndrome and shoe wear problems ${ }^{14}$.Closed Reduction decreases the chance of wound complications, infection \& much more others like wise in open reduction.

Many sources advocate ORIF (Open Reduction Internal Fixation) as the treatment of choice for most of intraarticular calcaneous fractures, but the complication rate remains significant ${ }^{15}$. Even with ORIF not all patients have well to excellent results and the outcome seems to be more related to the initial injury pattern as well as the surgeon's experience.

Other studies have found moderate correlations between the restoration of articular congruity and outcome. If the absolute articular restoration is not as important as once thought, other factors may play a more important role in outcome. Restoration of calcaneal width, varus/valgus alignment and pitch may be more influential than previously thought. In our series, despite the lack of articular reconstruction, the patients whose hindfoot alignment was restored appeared to experience a good result.

As our preliminary outcomes are promising, it may be that restoration of hindfoot alignment without an articular reconstruction may be an attractive alternative or compromise between formal ORIF and simple splinting in situ. Of cause the technique learning curve is slightly more and very long term results still warranted, the technique is relatively simple with proper skills, restores hindfoot alignment satisfactorily and with few complications.

\section{References}

[1] Crosby LA,Fitzgibbons TC.Open reduction \& Internal fixation of type 2 intraarticular calcaneus fractures. Foot Ankle Int. 1996; $17: 253-258$

[2] Essex-Lopresti P.The mechanism,reduction technique \& results in fractures of the os calcis.Br. J. Surg.1952; 39:395-419.

[3] Rammelt S, Grass R, Schikore H,Zwipp H.Injuries of the chopart joint. Unfallchirurg.2002; 105:371-383.

[4] Rammelt S, Gavlik JM, Barthel S,Zwipp H. The value of subtalar arthroscopy in the management of intraarticular calcaneus fractures. Foot Ankle Int. 2002; 23:906-916.

[5] Rammelt S, Barthel S, Biewener A, Gavlik JM, Zuipp H. Calcaneus fractures. Open reduction \& internal fixation. Zbl. Chir. 2003; 128:517-528.

[6] Gavlik JM,Rammelt S, Zwipp H. Percutaneus arthroscopically assisted osteosynthesis of calcaneus fractures. Arch Orthop. Trauma Surg. 2002; 33:63-71.

[7] Gavlik JM,Rammelt S, Zwipp H. The use of subtalar arthroscopy in open reduction \& internal fixation of intraarticular fixation of calcaneal fractures. Injury.2002; 33:63-71.

[8] Eastwood DM,Langkamer VG, Atkins RM. Intraarticular fractures of the calcaneum. Part 2: open reduction \& internal fixation by 
the extended lateral transcalcaneal approach. J Bone \& Joint Surg (Br) 1993; 75 B: 189-95.

[9] Bohler L. Behandlung der Fersenbeinbruche. Arch. Klin Chir. 1929; 157:723-732.

[10] Bohler L.Diadnosis,pathology and treatment of fractures of the os calcis. J. Bone Joint Surg. 1931; 13:75-89.

[11] Bohler L. Fersenbeinfrakturen. In: Bohler L, editor. Dir Technik der Knochenbruchbehandlung. Wien: Maudrich; 1957.p 2148-217.

[12] Gissane W.News notes: Proceedings of the British Orthopedic Association. J Bone Joint Surg. 1947; 29:254-255.

[13] Forgon M. Closed reduction \& percutaneous osteosynthesis: technique \& results in 265 calcaneal fractures. In: Tscherne H, Schatzker J, editors. Major fractures of the pilon, the talus \& the calcaneus. Berlin: Springer-Verlag; 1992.p.207-13

[14] Randle JA,Kredler HJ, Stephen D, et al. Should calcaneal fractures be treated surgically? Clin Orthop 2000; 377:217-27.

[15] Folk JW, Starr AJ, Early JS. Early wound complications of operative treatment of calcaneus fractures: analysis of 190 fractures. J Ortho. Trauma 1999; 13:369-72. 